\title{
CARACTERÍSTICAS DE LAS PISCINAS DE AIRE FRÍO (CAPS) EN EL INTERIOR SURESTE PENINSULAR
}

\author{
David Espín Sánchez ${ }^{(1)}$, Víctor Ruiz Álvarez ${ }^{(1)}$, Javier Martí Talavera ${ }^{(2)}$, Marcos Ruiz \\ Álvarez ${ }^{(3)}$, Juan Antonio Amor Jiménez ${ }^{(1)}$, Francisco Sandoval Gómez ${ }^{(4)}$, Miguel \\ Ángel Requena ${ }^{(1)}$, Pedro José Gómez Cascales ${ }^{(5)}$ y José María Serna García ${ }^{(6)}$ \\ (1) Departamento de Geografía (Universidad de Murcia), Calle Santo Cristo s/n, david.espin1@um.es \\ (2) Instituto Interuniversitario de Geografía (Universidad de Alicante), \\ Carretera de San Vicente del Raspeig, s/n, jmt25@alu.ua.es \\ (3) Instituto Universitario del Agua y Medio Ambiente (INUAMA). Universidad de Murcia. marcos.ruiz1@um.es \\ (4) Dipartamento di Ingegnieria civile, edile e ambientale (ICEA). Università degli Studi di Padova. \\ francisco.sandovalgomez@studenti.unipd.it \\ (5) SCOLAb, Universidad Miguel Hernández (Elche), p.gomez@umh.es \\ ${ }^{(6)}$ Licenciado en Geografía e Historia por la Universidad de Murcia
}

\section{Resumen:}

El interior sureste peninsular se caracteriza por presentar temperaturas mínimas inferiores $-15^{\circ} \mathrm{C}$ con relativa frecuencia, especialmente en las altiplanicies de la Sierra del Segura (Jaén). Las intensas y recurrentes inversiones térmicas en el área de estudio generan acumulaciones de aire frío en valles y cuencas montañosas, debido principalmente al gran número de días estables al año. Los episodios nocturnos de piscinas de aire frío (CAPs) que son identificados desde el 1 de diciembre 2016 al 28 de febrero de 2017 se clasifican según la tipología de Dorninger et al., (2011), atendiendo principalmente a la evolución de la temperatura durante las horas nocturnas en diferentes niveles altitudinales $(850-1770 \mathrm{~m})$. Se analizan varios episodios de piscinas frías permanentes durante varias jornadas consecutivas, constituyendo piscinas de aire frío persistentes (PCAPs). Los estudios llevados a cabo a través del análisis de sondeos atmosféricos, la red propia de datalogger, estaciones meteorológicas completas complementarias, y la cartografía sinóptica desde 1000 a $300 \mathrm{hPa}$, ha determinado que la nubosidad y los flujos intensos en niveles altos $(700 \mathrm{hPa})$ son los principales responsables para la rotura de los ciclos de vida de las CAPs, principalmente debido a circulaciones zonales y nubosidad asociada a dorsales subtropicales marítimas.

Palabras clave: inversión, piscina de aire frío, sondeos, datalogger

\section{Abstract:}

The peninsular southeast interior, is characterized by presenting values of minimum temperatures below $-15^{\circ} \mathrm{C}$ with relative frequency, especially in the plateaus of the Sierra del Segura. The intense and habitual thermal investments that are registered in the study area, generate cold air accumulations in valleys and mountain basins, mainly due to the large number of stable days per year. The nocturnal episodes of cold air pools (CAPs) that are identified from December 1, 2016 to February 28, 2017, are classified according to the typology of Dorninger et al., according to the evolution of the temperature during the night hours in different altitudinal levels. In addition, several episodes of permanent cold pools are analyzed over several consecutive days, constituting authentic pools of 
persistent cold air (PCAPs). The studies carried out, through the analysis of atmospheric soundings, the own datalogger network, complementary complete meteorological stations, and synoptic cartography from 100 to $300 \mathrm{hPa}$, have contributed to conclude that the cloudiness and the intense flows at high levels $(700 \mathrm{hPa})$ are the main responsible for breaking the life cycles of the CAPs.

Key words: inversion, cold air pools, sounding, datalogger

\section{Introducción}

Las piscinas de aire frío (CAP) son acumulaciones de aire frío en un sector topográfico deprimido, que suelen depositarse durante madrugadas estables (vientos flojos y cielos despejados). Después de 90 años de los primeros registros de temperatura en Gstettneralm (Austria), en los que se observaron temperaturas mínimas extremadamente bajas con elevada frecuencia (valores mínimos por debajo de $-50^{\circ} \mathrm{C}$ ) en los fondos de dolinas (Schmidt, 1930), se han llevado a cabo multitud de análisis de los ciclos de vida (formación, mantenimiento y rotura) de las CAPs en zonas de valle, análisis de procesos de inversión térmica, y observación de temperaturas mínimas en los fondos de dolinas.

$\mathrm{Al}$ centrar los estudios en época reciente, se establecen investigaciones entre el otoño 2001 y el verano de 2012, donde se estudian los mecanismos de formación y ruptura de piscinas de aire frío en las dolinas del Gstettneralm (Pospichal et al., 2003; Whiteman et al., 2004) y las de Peter Sinks en Utah (Clements et al., 2003). Concluyeron que, durante las noches despejadas, en el interior de las dolinas las tasas de enfriamiento son más importantes tras la puesta del sol, mientras que el enfriamiento es más gradual durante la noche y posterior madrugada. Además, el propio Whiteman et al. (2001), estudió dos eventos de PCAPs en la cuenca de Columbia, utilizando para ello modelos númericos de mesoescala (Zhong et al., 2001), así como el cráter experimental (METCRAX), donde se analizó la formación y destrucción de los procesos de inversión térmica (Whiteman 1990, Clements et al. 2003 y Steinacker et al., 2007).

Scherhag (1948), adopta una serie de criterios para la determinación de las CAPs: 1) Una baja cerrada con un mínimo de temperatura en la troposfera media y alta (300$700 \mathrm{hPa}$ ), 2) existencia de un campo de presión superficial poco definido, o lo que es lo mismo, presión atmosférica intermedia y un patrón de presión que no suele mostrar características marcadas, y 3) el espesor geopotencial de 500/1000 hPa, con intervalos de $40 \mathrm{gpm}$, debe mostrar al menos un contorno cerrado.

Las piscinas de aire frío, además, se han observado en zonas montañosas, donde se asocian, con frecuencia, a drenajes de aire frío por las laderas de valles. Se realizan varios estudios de observación de estos flujos a través de las cuencas en terreno montañoso, causados por un rápido enfriamiento de las CAPs en contacto con pendientes elevadas, que se aceleran por las laderas de valle al poseer una flotabilidad negativa (Barr y Orgill 1989; Neff y King 1989; Gudiksen et al., 1992; LeMone et al., 2003). Además, la rotura de las CAPs ha sido ampliamente estudiada con el análisis del crecimiento convectivo de la capa límite después del amanecer (Whiteman y McKee, 1982), con la erosión turbulenta en niveles superiores de la CAP (Petkovsek, 1992; Vrhovec y Hrabar 1996) por advecciones de aire frío (Zhong et al., 2001), drenaje de aire de los valles (Zängl, 2002), 
ligados al paso de frentes (Whiteman et al., 2001), o finalmente con erosión turbulenta de la piscina Zhong et al., 2003; Fritts et al., 2010).

Tal y como pretende analizar el presente estudio, durante las noches estables de marcada estratificación, se generan importantes diferencias de temperatura entre el fondo de valles y dolinas, y zonas de exterior más elevadas, llegando a suponer varias decenas de grados en un desnivel altitudinal muy escaso (Clements et al., 2003) y Zängl, 2005).

Recientemente, Miró et al., (2010) estudian las acumulaciones de aire frío en el valle del Segre y la Cerdenya catalana. En este mismo ámbito geográfico se identificaron CAPs mediante estudios de cuatro años de duración, simulación de mesoescala de alta resolución, y mediante una campaña experimental que se llevó a cabo en octubre de 2015 con el objetivo de encontrar evidencia experimental (Martínez et al., 2016). El equilibrio y la profundidad de la PAC es objeto de estudio mediante la relación con las ondas Kelvin-Helmholtz en Salk Lake City, (Utah) (Lareau y Horel, 2015). Por su parte, Burns, y Chemel (2014) analizan el modelo de "Weather Research and Forecasting" en valles alpinos de $10 \mathrm{~km}$, analizando los mecanismos clave de forzamiento de aire frío en las CAP.

El presente estudio pretende analizar los diferentes tipos de CAPs en el interior sureste peninsular durante la época invernal, determinando cuáles son las características de la dirección y velocidad de viento, humedad relativa, temperaturas, intensidad y amplitud de inversión, nubosidad y presión atmosférica superficial. Así mismo, se determinan las configuraciones sinópticas más comunes en la configuración de las CAPs y de las PCAPs.

\section{2 Área de estudio}

La zona de estudio tratada se ubica en el interior del sureste peninsular, más concretamente en el nodo interprovincial que une las provincias de Albacete (Castilla-La Mancha), Almería, Granada, Jaén (Andalucía) y Región de Murcia. Cuenta con una superficie de $4.720 \mathrm{Km}^{2}$ repartida entre las demarcaciones hidrográficas del Segura y del Guadalquivir. Está enclavada dentro de la cordillera Bética, encontrándose las mayores elevaciones en la mitad septentrional con altitudes que superan los $2000 \mathrm{msnm}$, como es el caso de La Sagra (2378 m.), Tornajuelos (2136 m.) y Las Cabras (2083 mm). El clima dentro de este ámbito se caracteriza por una distinguida heterogeneidad debido principalmente a la influencia que ejerce el relieve sobre los frentes atlánticos, registrándose unas medias de precipitación y de temperatura que pueden ir desde los $1300 \mathrm{~mm}$ y $9{ }^{\circ} \mathrm{C}$, en la parte más occidental, a los $350 \mathrm{~mm}$ y $16{ }^{\circ} \mathrm{C}$ en el extremo oriental del área de estudio.

Especial mención, debido a su singularidad, requiere los Campos de Hernán Perea situados dentro del Parque natural de las Sierras de Cazorla, Segura y Las Villas. Ubicados entre los municipios de Cazorla y Santiago-Pontones constituye la mayor altiplanicie kárstica de España, con una superficie de $144 \mathrm{Km}^{2}$. Su altitud media, cercana a los 1700 msnm, y su aislamiento, pues se encuentra circundada por elevaciones montañosas que superan los 2000 msnm, le confieren unas características climáticas únicas, representadas especialmente por unas temperaturas muy bajas. 


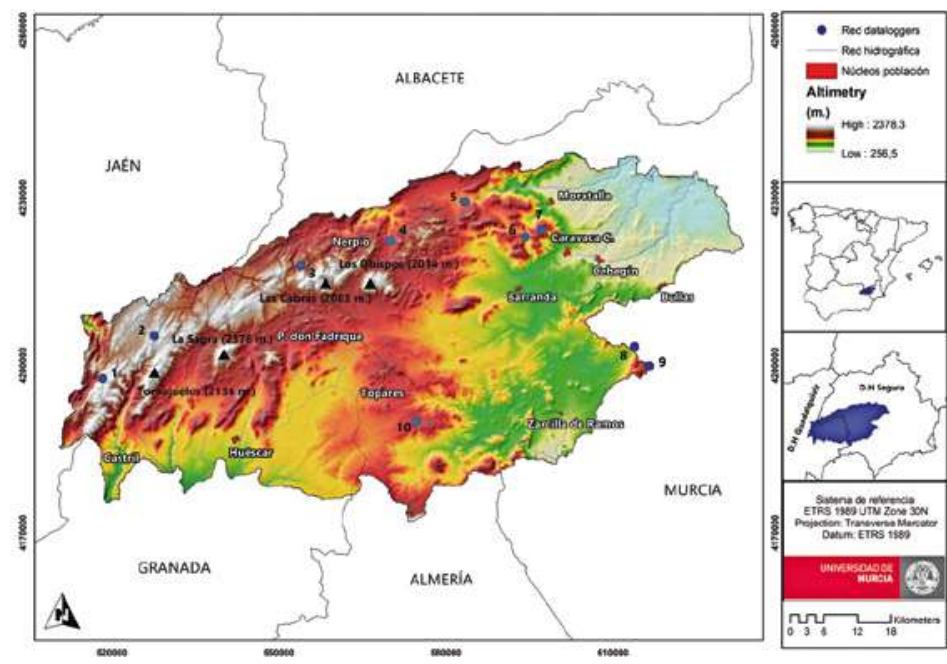

Fig. 1 Área de estudio

\section{Datos}

La obtención de los datos de campo del estudio se logra mediante la instalación de diez medidores de temperatura con datalogger incorporado, modelo Microlite II USB $32 \mathrm{~K}$ (32.000 registros). Posee una frecuencia de medición cincominutal, y un rango temporal de 90 días ( 1 de noviembre de 2016 al 31 de mayo de 2017). Los sensores presentan una precisión $\pm 0,3^{\circ} \mathrm{C}$, con una resolución térmica de $0,03^{\circ} \mathrm{C}$ (Figura 1 y Tabla 1 ). Dos de los sensores (Monterilla y Navalasno) han sido calibrados en el Centro Meteorológico Territorial (CMT) de Guadalupe por AEMET Murcia, siendo comparados con los termómetros oficiales en el interior de las garitas de AEMET durante dos semanas (con una desviación final de $+0,2{ }^{\circ} \mathrm{C}$ en las temperaturas mínimas, y que es tenido en cuenta en los resultados expuestos). Para la protección de radiación de los datalogger se utilizan ocho garitas Davis no aspiradas de siete platos (modelo 7714), situadas a una altura de 1,5 m., y dos garitas de tipo pagoda cedidas por AEMET Murcia, estas últimas utilizadas en Monterilla y Navalasno. Se sitúan en esta ocasión a $2 \mathrm{~m}$ de altura para evitar la acumulación de nieve que se almacena en los fondos de dolinas durante la época invernal (Figura 2). Las altitudes exactas de los sensores se muestran en la Tabla 1, donde se pueden obtener más detalles sobre la ubicación y precisión de los sensores.

Los datos meteorológicos de apoyo provienen de la estación meteorológica automática del Observatorio Astronómico de Calar Alto (Almería), a una altitud de $2167 \mathrm{~m}$ de altitud, al sur del área de estudio (provincia de Almería). Además, se utilizan las estaciones meteorológicas del Sistema de Información Agroclimático para el Regadío (SIAR) de la Puebla don Fadrique (Granada) y Casas del Rey-Moratalla (Región de Murcia). Los datos operan con una resolución horaria, incorporando datos de temperatura, velocidad y dirección del viento, así como registros de humedad relativa. Por su parte, la información 
meteorológica sinóptica para el análisis de cada uno de los eventos fue tomada por los reanálisis del Modelo del Centro Europeo (ECMWF) de Meteociel, y del Global Forecast System (GFS) de la NOAA, para los meses de diciembre, enero y febrero 2016-2017 (en línea en https://www.esrl.noaa.gov/psd/forecasts/reforecast2/wx_maps/index.html/ y http://www.meteociel.fr/modeles/archives/). Además, se recurre al reanálisis de la trayectoria de las masas de aire a través del HYSPLIT de la NOAA, a $1500 \mathrm{~m}$ de altitud.

\begin{tabular}{|c|c|c|c|c|c|}
\hline Observatorio & Punto & Altitud (m) & Provincia & Municipio & Sky View Factor \\
\hline Navalasno & 1 & 1770 & Jaén & Cazorla & 0,75 \\
\hline Monterilla & 2 & 1660 & Jaén & Santiago-Pontones & 0,91 \\
\hline Cañadas de Nerpio & 3 & 1439 & Albacete & Nerpio & 0,17 \\
\hline La Rogativa & 4 & 1152 & Murcia & Moratalla & 0,33 \\
\hline La Risca & 5 & 1047 & Murcia & Moratalla & 0,88 \\
\hline Gollaín & 6 & 1021 & Murcia & Caravaca de la Cruz & 0,60 \\
\hline Nevazo de Arriba & 7 & 1181 & Murcia & Caravaca de la Cruz & 0,80 \\
\hline Bco, del Cenajo & 8 & 870 & Murcia & Cehegín & 0,45 \\
\hline La Selva & 9 & 1521 & Murcia & Lorca & $1,00^{*}$ \\
\hline Cañadas de Cañepla & 10 & 1057 & Almería & María & 0,97 \\
\hline
\end{tabular}

Tabla 1.- Características de los datalogger utilizados en el área de estudio. *Estación situada en pico montañoso.

Por su parte, para el estudio de la presión atmosférica superficial se recurre al observatorio meteorológico de Caravaca de la Cruz (AEMET), anotándose los registros a las 06 h UTC, por situarse dentro del área de estudio. Para el análisis de la nubosidad, se recurre, por un lado, al observatorio meteorológico de 1er orden del Centro Meteorológico Territorial de Guadalupe-Murcia (AEMET) con los datos de octas de nubosidad a las $06 \mathrm{~h}$ UTC, y por otro, al reanálisis de la nubosidad del modelo ARPEGE $0,1^{\circ}$ a través de la http://web meteociel.fr. Finalmente, la superficie nevada, se estima mediante el producto (SMAP_L4_Snow_Mass) en $\mathrm{kg} / \mathrm{m}^{2}$.

Los sondeos atmosféricos utilizados son lanzados desde el CMT de Guadalupe Murcia (AEMET) a las 00 UTC desde el 1 de diciembre de 2016 al 28 de febrero de

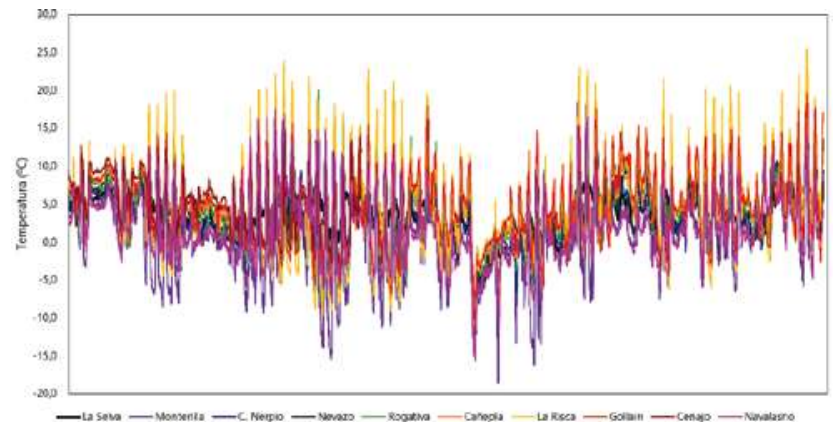

Fig. 2 Evolución de la temperatura en los diferentes datalogger del área de estudio (datos cada 20 minutos). Del 1 de diciembre 2016 al 28 de marzo 2017. 
2017. Se estudian, para cada nivel altitudinal correspondiente con los diez datalogger, valores de temperaturas, humedad relativa, dirección y velocidad de viento. Las temperaturas registradas, aquellas en "atmósfera libre", es decir, las que no están influidas por la topografía ni por ningún elemento que pueda distorsionar dichas temperaturas, son comparadas con los valores mínimos resultantes de los datalogger, para identificar la diferencia, o intensidad de inversión para ámbito geográfico.

\section{Métodos}

Los datos de temperatura veinteminutales de los datalogger utilizados, han servido para identificar las diferentes piscinas de aire frío nocturno (1 diciembre 2016 - 28 febrero 2017) durante el invierno meteorológico 16/17, atendiendo a la tipología de Dorninger et al., (2011), el cual establece ocho tipos de CAP según la evolución de la temperatura nocturna. Una CAP viene definida por la acumulación de aire frío en una depresion del terreno, principalmente durante la noche, y sujeto a condiciones de estabilidad (cielos despejados, altas presiones y vientos flojos). Además, se identifican algunos episodios de piscinas de aire frío persistentes (PCAPs), aquellas donde la temperature de los fondos de valle o depresiones permanecen por debajo de las registradas en altitudes superiores durante varios días (Whiteman et al., 2001). Los datos meteorológicos de las estaciones meteorológicas complementarias son utilizados para el análisis de las madrugadas con CAPs, con promedios de viento y humedad relativa de 0 a 8 UTC de la madrugada. Además, las retrotrayectorias de las masas de aire están calculadas en las 240 horas anteriores, cuyo destino se ubica en la altiplanicie de Hernán Perea a $1500 \mathrm{~m}$ de altitud. Finalmente, se propone un índice de tasa de descenso, con el fin de establecer el descenso de la temperatura $\left({ }^{\circ} \mathrm{C}\right.$ ) entre las 16:00 h (con insolación) y las 19:00 h UTC (nocturno con irradiación). Se pretende cuantificar los desplomes térmicos más intensos durante los primeros momentos de las CAPs (tras la puesta de sol).

\section{Eventos de piscinas de aire frío}

Se identifican las jornadas con piscinas de aire frío nocturnas (CAPs) en el área de estudio, mediante la utilización de las series de temperaturas de 10 datalogger de temperatura. De un total de 90 jornadas analizadas, en un total de 39 se identifican CAP nocturnas, lo que puede parecer un valor relativamente bajo, pero es preciso tener en cuenta que los meses de diciembre y enero fueron realmente inestables en el sureste peninsular (Espín Sánchez et al., 2017). Por tanto, durante el invierno 16/17 el $43,3 \%$ de las madrugadas se caracterizan por presentar aire frío en los valles y cubetas interiores donde se encuentran instalados los datalogger. El tipo de CAP más común corresponde a la de erosión turbulenta (33\%), confirmando que el invierno analizado estuvo sujeto a una gran dinámica atmosférica. En ese sentido, los dos tipos de CAP de formación temprana y perturbación baja no se representan en el área de estudio (Tabla 2). Por meses, diciembre, debido principalmente a su condición anticiclónica, representa el $42 \%$ de las CAP, mientras que enero (33\%) y febrero (25\%) presentan menos protagonismo. 


\section{Tipode Piscina Descripción Fechas $\quad N^{\circ}$ Eventos (\%)CAPs}

1 Evolución inalterada 11-dic/12-dic/01-ene/02-ene

\begin{tabular}{|c|c|c|c|c|}
\hline & & /25-ene/18-feb/27-feb & 7 & 17,9 \\
\hline 2 & Formación tardía & 02-dic/10-dic/24-dic/18-ene/14-feb & 5 & 12,8 \\
\hline 3 & Rotura temprana & $*$ & 0 & 0,0 \\
\hline 4 & Evento de mezcla & 07-dic/16-ene/01-feb & 3 & 7,7 \\
\hline \multirow[t]{2}{*}{5} & Perturbación alta & 06-dic/13-dic/26-dic/27-dic & & \\
\hline & & /28-dic/30-dic/31-ene/13-feb/22-feb & 9 & 23,1 \\
\hline 6 & Perturbación baja & $*$ & 0 & 0,0 \\
\hline 7 & Erosión turbulenta & 03-dic/22-dic/25-dic/31-dic/03-ene/0 & & \\
\hline
\end{tabular}

$8 \quad 09$-ene/10-ene/12-ene/02-feb/10-feb/25-feb/26-feb $13 \quad 33,3$

8 Ventana de inversión 21-ene/23-ene $\quad 2 \quad 5,1$

$9 \quad$ Piscinas de aire frío $25-27 \mathrm{dic} / 30 \mathrm{dic}-3 \mathrm{ene} / \quad 5$

persistentes (PCAPs) 7-8 ene/2-4 feb/27-28 feb.

Total

Tabla 2.- Categorización de los eventos de piscina de aire frío por jornadas (1 diciembre 2016 - 28 febrero 2017)

\section{a) Evolución inalterada}

Con un total de 7 eventos de CAPs del tipo evolución inalterada, este tipo de piscina fría es la más común en la literatura científica, si bien las citadas condiciones inestables anteriormente citadas, generó un escaso número de madrugadas con inversiones térmicas "limpias". Durante las primeras horas de la tarde se registran tasas de enfriamiento muy significativas, además, con un inicio medio de la constitución de la CAP de las 17:43 h UTC (aproximadamente 45 minutos después del ocaso) (Figura 2A). En el análisis de la tasa de descenso de temperatura desde las 17 a las 20 horas UTC en los diferentes datalogger instalados, el tipo 1 de evolución inalterada es el que registra el valor más elevado $\left(4,9^{\circ} \mathrm{C}\right)$, con registros absolutos en Monterilla de $13,4^{\circ} \mathrm{C}$ durante la jornada del 25 de enero 2017. El tipo de CAP con el segundo valor más elevado (formación tardía) obtiene registros medios de $4,3^{\circ} \mathrm{C}$. En contraposición, la ventana de inversión, perturbación alta o evento de mezcla registran tasas inferiores a $3,5^{\circ} \mathrm{C}$.

El flujo medio de viento nocturno que arrojan los sondeos es de a $19,6 \mathrm{~km} / \mathrm{h}$, con velocidades medias más elevadas entre $1100-1200 \mathrm{~m}$ de altitud $(>21 \mathrm{~km} / \mathrm{h})$. Mientras, en niveles más elevados $(>1500 \mathrm{~m}$ ) la velocidad media desciende a $18 \mathrm{~km} / \mathrm{h}$, lo que favorece los procesos de inversión térmica en Navalasno y Monterilla, donde las temperaturas mínimas son las más bajas del área de estudio. En efecto, las velocidades medias arrojadas por las estaciones meteorológicas superficiales arrojan entre 2 y $4,5 \mathrm{~km} / \mathrm{h}$ (Casas del Rey y Puebla don Fadrique respectivamente), donde se advierte una velocidad menor que en atmósfera libre, debido a la influencia de la topografía. La dirección media del viento durante las noches de piscina inalterada varía entre 216 y $236^{\circ}$ (componente sursuoreste), a excepción de altitudes cercanas a $1500 \mathrm{~m}$, con componente sur.

Por su parte, los porcentajes de humedad relativa media llegan al 63\%, con una clara dicotomía entre niveles más bajos (850-1450 m), con valores entorno al 70\%, siendo 
entre el 77 a 93\% en los observatorios superficiales, mientras que en niveles superiores (>1600 m) los porcentajes se sitúan entre el 45 y el $39 \%$, con valores medios de $52,1 \%$ en Calar Alto. El bajo punto de rocío vuelve a favorecer los procesos de I.T y formación de CAP en niveles superiores (altiplano de Hernán Perea con Navalasno y Monterilla). La humedad relativa a $700 \mathrm{hPa}$ registra porcentajes medios de $15,4 \%$, sin duda, el valor más bajo de todos los tipos de piscina analizadas, y un claro indicador de sequedad en niveles medios atmosféricos a la hora de configurarse episodios de piscinas frías inalteradas y procesos intensos de inversión térmica.

Además, las intensidades de inversión térmica son relativamente importantes $\left(-10,2^{\circ} \mathrm{C}\right)$, registrando uno de los valores más elevados de todos los tipos de piscina analizados. En ese sentido, Monterilla $\left(-14,3^{\circ} \mathrm{C}\right)$ con clara supremacía, La Risca $\left(-12,8^{\circ} \mathrm{C}\right)$ y La Rogativa $\left(-11,7{ }^{\circ} \mathrm{C}\right)$ registran los procesos de I.T más vigorosos del área de estudio.

La nubosidad apenas tiene influencia, anotándose una media de 1,6 octas de nubosidad en el observatorio meteorológico de Murcia, mientras que tras el reanálisis del modelo ARPEGE no advierte nubosidad en la Sierra de Segura durante las jornadas analizadas $(0,3)$. Esto da lugar, a que la amplitud térmica media más elevada se registre en las jornadas con estabilidad atmosférica $\left(11,1^{\circ} \mathrm{C}\right.$ de diferencia entre Monterilla con $-9,4{ }^{\circ} \mathrm{C}$ y La Selva con 1,7), en apenas $140 \mathrm{~m}$. de desnivel. Finalmente, se advierte una importante correlación entre una mayor amplitud de inversión y temperaturas mínimas más bajas en Monterilla $(0,8)$, Cañadas de Nerpio, Rogativa y La Risca $(0,6)$.

Por último, éste tipo de piscinas, denominadas "perfectas" en la literatura científica por Clements et al.,2013; Whiteman et al., 2004, y Dorninger et al., 2011, se producen con situaciones sinópticas marcadas por una potente dorsal de origen subtropical marítimo a al oeste de la Península, con altas presiones centradas. Mientras, las altas presiones son protagonistas en el área de estudio, con un valor medio de 1022,4 $\mathrm{hPa}$. La rotura media de la piscina se produce a las 9:09 h UTC, aproximadamente una hora después de la salida del sol, produciéndose, pot tanto, un calentamiento muy rápido en todas las estaciones en las 2,5 h siguientes a la salida del sol.

\section{b) Formación tardía}

Un total de 5 eventos conforman el tipo 2 o de formación tardía de CAP en el área de estudio, cuya característica principal es la de la constitución de la piscina de aire fría durante primeras horas de la madrugada (3:05 h UTC). Por consiguiente, tras su rotura a las 9:05 h UTC, constituye un tipo de piscina fría de corta duración (de unas 6 horas). Estos episodios de eventos tardíos ocurren habitualmente cuando la nubosidad se disipa o los vientos se debilitan en las depresiones montañosas, por ejemplo, después del paso nocturno de frentes. A pesar de su corta duración, suelen dar lugar a inversiones térmicas potentes, tal y como lo demuestra los $-8,1^{\circ} \mathrm{C}$ de intensidad media en el área de estudio. En ésta ocasión, Monterilla, La Risca, Gollaín y la Rogativa registran los valores más importantes, siendo relativamente parecidas entre $-10,5$ y $-9,5^{\circ} \mathrm{C}$. Además, durante los episodios analizados se registran las temperaturas (sondeos) en niveles medios-bajos atmosféricos más bajas de todos los tipos de CAP, con un promedio de $5,3^{\circ} \mathrm{C}$, lo que 
evidencia episodios fríos durante la formación de las piscinas tardías (Figura 2b). La amplitud térmica de inversión es de $8,3^{\circ} \mathrm{C}$ entre Monterilla $\left(-7,6^{\circ} \mathrm{C}\right)$ y La Selva $\left(0,7^{\circ} \mathrm{C}\right)$

Durante estas jornadas, la humedad relativa media del sondeo es elevada, entorno al $69 \%$, con los valores más importantes en niveles más altos (>1500 m), superiores al 76\%, lo que puede explicar que en ésta ocasión los valores mínimos de temperatura no sean tan bajos en Navalasno y Monterilla como en el tipo 1. En los observatorios complementarios, se observan humedades relativas próximas a la saturación en 4 de los 5 eventos, incluso en el observatorio de Calar Alto (2167 m, donde tan sólo el 24 de diciembre de 2016 la humedad relativa media durante la madrugada es del $45 \%$. La HR a $700 \mathrm{hPa}$ es igualmente baja, con una media del $41,8 \%$. Por lo general, se mantiene baja en episodios de piscina de aire frío.

Por su parte, la intensidad del flujo nocturno es curiosamente la más baja de todos los tipos de CAP, con una velocidad media de $13,4 \mathrm{~km} / \mathrm{h}$ según el sondeo, lo que puede llevar a la conclusión de que la formación tardía obedece más bien a la rotura de nubosidad que a la influencia del viento para la constitución de dichas piscinas. Efectivamente, los valores medios de viento en los observatorios de Casas del Rey la Puebla don Fadrique se sitúan entre 3 y $7 \mathrm{~km} / \mathrm{h}$., mientras que, en Calar Alto, la velocidad se sitúa entre 10 y $21 \mathrm{~km} / \mathrm{h}$ (elevada para romper el estrato de inversión). La dirección media del flujo es de componente sur (media de 156,3 $3^{\circ}$, con componente sureste entre 1000-1100 m de altitud.

La presión atmosférica media sigue siendo elevada, con 1024,3 hPa, la más importante de los eventos de piscina analizados, lo que repercute en que la nubosidad sea poco significativa en general (2,5 octas de nubosidad). La configuración sinóptica viene marcada por la irrup-

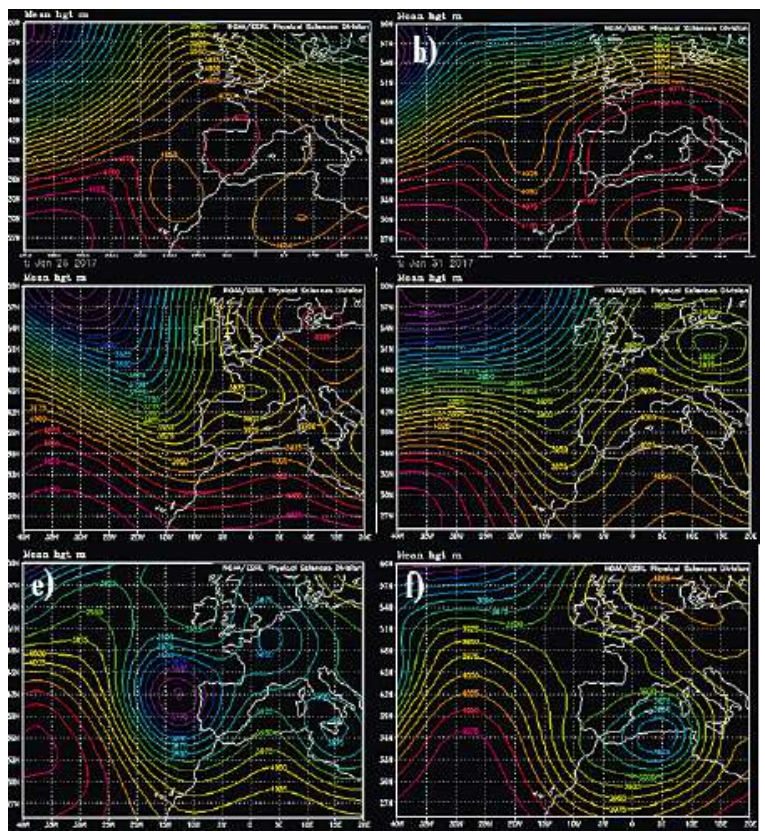
ción de una potente dorsal de origen subtropical marítimo. Se genera un claro patrón de nubosidad a primeras horas de la madrugada en 4 de las 5 situaciones analizadas, que posteriormente se va disipando. Sólo en una de ellas, el viento en niveles altos (18 de enero) fue el responsable de la formación de la piscina tardía, debido a la presencia de una BFA sobre el Mediterráneo, que generó vientos flojos durante gran parte de la madrugada.

Fig. 3 Patrones sinópticos más frecuentes según las diferentes categorías de CAP de Dorninger: a) evolución inalterada, b) formación tardía, c) evento de mezcla, d) perturbación alta, e) erosión turbulenta, y f) ventana de inversión. 


\section{c) Evento de mezcla}

Los fuertes vientos causan con frecuencia importantes erosiones en las piscinas de aire frío. Cuando los vientos por encima del estrato de inversión son lo suficientemente importantes, los remolinos turbulentos mezclan el aire más caliente de la parte superior de la inversión, lo que provoca un aumento de las temperaturas en niveles inferiores. Según Dorninger, los parámetros más influyentes para su configuración son los siguientes: 1) cizalladura vertical de vientos horizontales, 2) debilidades de las partículas generadas por esta cizalladura, 3) interacciones con la topografía más elevada de alrededor, y 4) la estabilidad dentro del conjunto de aire frío.

Tres de estos eventos se observaron durante el período de observación, concretamente las jornadas del 7 de diciembre de 2016, 16 de enero de 2017, y el 1 de febrero de 2017, siendo, por tanto, uno de los tipos de inversión menos comunes, y que con más complejidad ha supuesto en el mundo científico para explicar su formación.

La formación de la piscina es tardía, a las 20:56 horas UTC, con una duración media de 12 horas y 7 minutos. Sin embargo, la destrucción de la CAP no observa grandes diferencias con el resto de piscinas, siguiendo el patrón habitual (9:05 h UTC). En general, los valores de humedad relativa del sondeo son relativamente bajos, especialmente en cotas menos elevadas ( $46 \%$ a unos $850 \mathrm{~m}$ de altitud), así como en niveles superiores (>1600 m) rondando el 50\%. Tal y como se observa en las tipologías anteriores, los valores higrométricos en niveles altos $(700 \mathrm{hPa})$ son relativamente bajos, con un promedio del 32\% (Figura 2c). Existe cierta relación entre los bajos valores higrométricos a 700 hPa y los valores más bajos de temperaturas mínimas en los parajes del Nevazo, Risca y Gollaín $(0,47)$. A nivel superficial, los valores higrométricos son relativamente elevados $(>70 \%)$, si bien es cierto que durante las madrugadas analizadas se producen importantes alteraciones en los valores.

Por su parte, los flujos nocturnos (sondeos) son los más elevados de todas las tipologías, confirmando que el viento tiene un papel fundamental a la hora de la configuración de los tipos "evento de mezcla", con una posible teoría sobre su génesis precediendo la entrada de frentes atlánticos. Los valores medios llegan a los $28 \mathrm{~km} / \mathrm{h}$., siendo superiores por encima de $1700 \mathrm{~m}(30,6 \mathrm{~km} / \mathrm{h})$ con una marcada componente este $\left(90-95^{\circ}\right)$. En el resto de rangos altitudinales, principalmente entre 850 y $1200 \mathrm{~m}$ el flujo predominante es de componente sur-suroeste $\left(180-190^{\circ}\right)$, con velocidades que por lo general se sitúan sobre los $27 \mathrm{~km} / \mathrm{h}$. En los observatorios de superficie, tanto la Puebla don Fadrique como Casas del Rey $(1200 \mathrm{~m})$ registran velocidades muy bajas $(3,9$ y $4,3 \mathrm{~km} / \mathrm{h})$, mientras que en niveles superiores $(>2100 \mathrm{~m}$ ), la velocidad media es de $21,3 \mathrm{~km} / \mathrm{h}$ Incluso en niveles más bajos, durante las madrugadas analizadas, se producen aceleraciones puntuales del viento, lo que da lugar a momentáneas rupturas de la inversión.

Finalmente, éste tipo de CAP da lugar a intensidades de inversión más modestas que el resto de tipos $\left(-8,0^{\circ} \mathrm{C}\right)$, mientras, que la amplitud de inversión también es de las más bajas de todos los tipos analizados, con diferencias de $6,8^{\circ} \mathrm{C}$ entre los $-4,5^{\circ} \mathrm{C}$ de Monterilla y $2,3^{\circ} \mathrm{C}$ de La Selva. La nubosidad parece no influir para nada durante las jornadas analizadas, con una media de 1,0 octa en Murcia, sin embargo, sí existe retención en la Sierra del Segura, con un promedio de 4,4 octas. Con una presión 
atmosférica de $1023 \mathrm{hPa}$, las altas presiones superficiales son dominantes, aunque en dos de las tres jornadas analizadas la península ibérica se encontraba afectada por dos vaguadas polares de onda larga, poco activa. Generan nubosidad, en general de tipo alto, y un flujo del noroeste no demasiado importante. Por tanto, ligera inestabilidad que en momentos llega a romper la IT, aunque no del todo.

\section{d) Perturbación alta}

Las perturbaciones en la parte superior de la piscina de aire frío muestran relación con los vientos existentes por encima de la inversión, como se demostró para el caso de la "mezcla". En la parte superior del área de estudio se observan irrupciones de masas de aire asociado a vientos moderados, aunque no lo suficientemente importantes como para romper las piscinas de aire frío de niveles inferiores. En total son 9 los eventos identificados, lo que supone el 16,7\% de las CAP reconocidas, sin duda un evento bastante reconocible en el interior sureste peninsular. Tal y como se aprecia en la figura $5 \mathrm{~d}$, las trayectorias de las masas de aire provienen principalmente del océano Atlántico, siguiendo un flujo atmosférico del oeste, asociado a perturbaciones atlánticas.

Los valores de humedad relativa son relativamente bajos (50,4\%), constituyendo la media más baja de los episodios analizados. Sin duda, especial atención merecen los valores de niveles más elevados, precisamente donde las CAP son más fácilmente destruidas, con valores por debajo del $47 \%$ a partir de $1300 \mathrm{~m}$ de altitud. En efecto, el observatorio de Calar Alto promedia un valor de 49,4\%, corroborando que en niveles más elevados $(>2000 \mathrm{~m})$ el flujo de componente sur presenta poca carga higrométrica. Éste flujo de componente sur $\left(181^{\circ}\right)$, según el sondeo, adquiere velocidades más importantes en dichos niveles más elevados, pues por encima de $1500 \mathrm{~m}$ de altitud registra velocidades de más de $20 \mathrm{~km} / \mathrm{h}$ (Calar Alto con 24,4 km/h, el más elevado de todos los tipos), mientras que, en niveles más bajos, apenas se superan los $15-16 \mathrm{~km} / \mathrm{h}$, concluyendo, que, los marcados flujos de viento en niveles altos son los responsables, principalmente, de romper parcialmente las piscinas de aire frío en sus cotas más elevadas. Máxime, cuando se pone de manifiesto que los observatorios que rondan los $1200 \mathrm{~m}$ de altitud

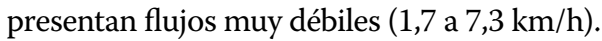

A pesar, que la amplitud de inversión sea una de las más bajas de todos los tipos analizados (las temperaturas mínimas tienden a igualarse), sí que se aprecia una marcada intensidad de la inversión térmica $\left(-10,2^{\circ} \mathrm{C}\right)$. Y es que existe una relación notable entre intensidades de inversión elevadas y temperaturas mínimas más bajas, como por ejemplo en La Risca $(0,76)$, Monterilla $(0,75)$ o La Rogativa $(0,65)$. Todo ello a pesar que la nubosidad también juega un papel importante, con una media de 5,6 octas en el observatorio de AEMET de Murcia, a pesar de que la estabilidad es manifiesta, con una presión atmosférica media de 1024,1 hPa. En cambio, la nubosidad en la Sierra del Segura $(2,9)$ presenta menos incidencia, corroborando que es el viento el responsable principal de la erosión de las CAP en los niveles más elevados.

Finalmente, es preciso añadir, que se trata del tipo de piscina más duradera, con un total de 16 horas y 11 minutos, constituyéndose a las 17:01 h UTC (muy temprana), y rompiéndose a las 9:12 h UTC. El patrón sinóptico principal viene definido por dos 
VARIABILIDAD Y CAMBIO CLIMÁTICO

Fig. 4. Ejemplos de piscinas de aire frío (CAP) según las diferentes categorías de CAP de Dorninger: a) evolución inalterada, b) formación tardía, c) evento de mezcla, d) perturbación alta, e) erosión turbulenta, y f) ventana de inversión.
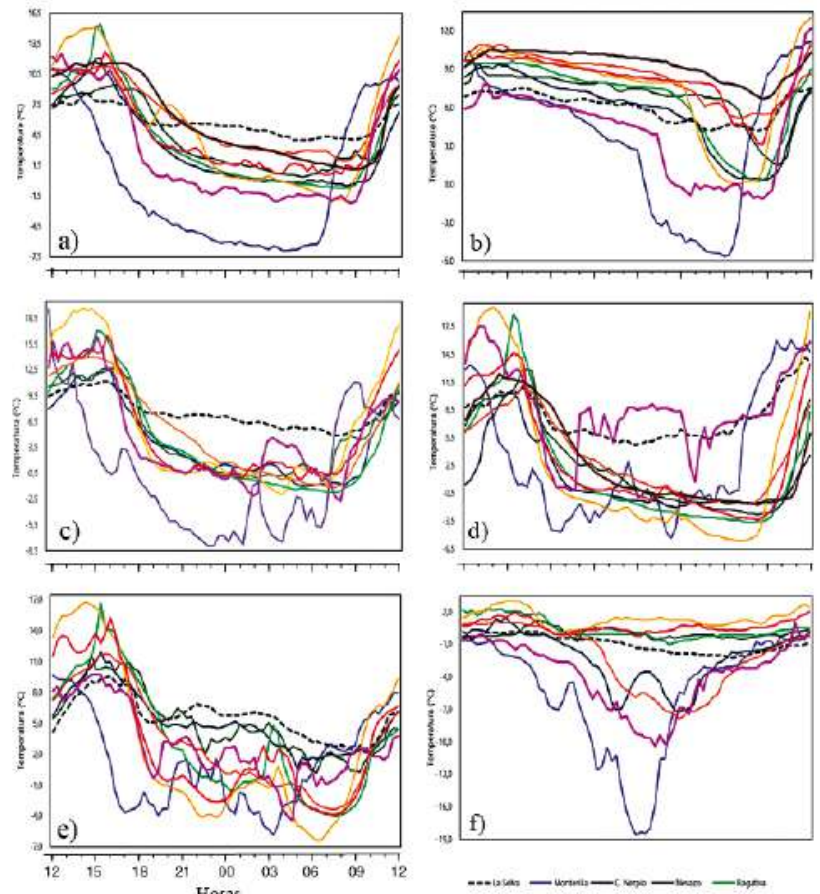

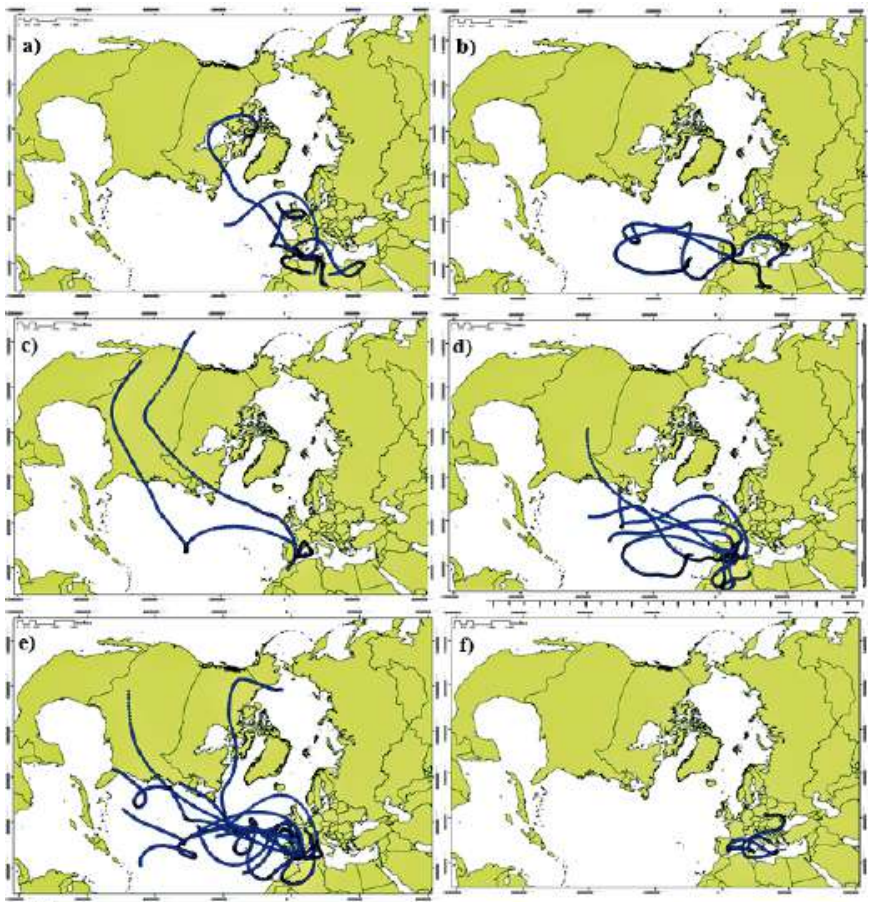

Fig. 5. Trayectorias de las masas de aire a $1500 \mathrm{~m}$ de altitud según jornadas por categorías de CAP de Dorninger: a) evolución inalterada, b) formación tardía, c) evento de mezcla, d) perturbación alta, e) erosión turbulenta, y f) ventana de inversión. 
claras situaciones. Por un lado, la dorsal subtropical al suroeste de la península ibérica, con altas presiones centroeuropeas $(1024,1 \mathrm{hPa})$, que generan un flujo débil a $700 \mathrm{hPa}$, y nula nubosidad. Por otro lado, perturbaciones atlánticas que se posicionan al oeste de la Península, cuyas vaguadas generan un flujo moderado en niveles altos y nubosidad, en varias ocasiones con valores superiores a 5 octas.

\section{e) Erosión turbulenta}

La erosión turbulenta de una piscina de aire frío es un proceso de extracción lento en el que la parte superior de la piscina de aire frío va erosionando capa por capa. Investigaciones anteriores sugieren que los vientos por encima de la inversión deben aumentar de velocidad para continuar el proceso de erosión (Petkovsek 1992; Rakovec y otros 2002, Zhong et al., 2003). A pesar de que en la literatura científica son poco comunes, se identifican 13 jornadas, constituyendo el tipo de CAP más común en el área de estudio (28,7\%).

Tal y como ocurre en el tipo de CAP de perturbación alta, la intensidad del viento en el sondeo es superior en niveles altos, arrojando valores superiores a los $15 \mathrm{~km} / \mathrm{h}$ en altitudes por encima de $1500 \mathrm{~m}$ de altitud. Este flujo, algo más intenso, tiene una componente sur-sureste $\left(162,1^{\circ}\right)$, mientras que, en niveles inferiores, donde las CAP suelen aguantar mejor, la velocidad media es relativamente baja $(12,4 \mathrm{~km} / \mathrm{m})$, predominando igualmente la componente sur-sureste $\left(165,3^{\circ}\right)$. Concretamente los valores medios de viento en Calar Alto logran superar los $20 \mathrm{~km} / \mathrm{h}$. durante 6 madrugadas analizadas, mientras que difícilmente sobrepasan los $8 \mathrm{~km} / \mathrm{h}$. tanto en Casas del Rey o la Puebla donde Fadrique $(1200 \mathrm{~m})$. Ello repercute en los valores de humedad relativa aportados por el sondeo, que son relativamente bajos, entorno al $57 \%$, aunque con importantes diferencias, un marcado flujo más seco en altitudes superiores a $1500 \mathrm{~m}$ de altitud $(43,1 \%)$, y valores realmente destacables entre 850 y $1300 \mathrm{~m}$. (cercanos al 70\%). Esto se corrobora con los datos en superficie, ya que los observatorios situados sobre los $1200 \mathrm{~m}$ obtienen promedios entre el 90-99\%, mientras que, en Calar Alto en niveles superiores, hasta en 7 ocasiones los valores higrométricos se sitúan por debajo del $50 \%$, con jornadas de $15 \%$ (31 de diciembre).

Varios factores ayudan a que la intensidad de la inversión durante dichos episodios sea realmente destacable $\left(-9,8^{\circ} \mathrm{C}\right)$, con una amplitud de inversión igualmente destacable $\left(9,3^{\circ} \mathrm{C}\right)$ entre la media de las mínimas de Monterilla $\left(-6,6^{\circ} \mathrm{C}\right)$ y la de la Selva $\left(2,7^{\circ} \mathrm{C}\right)$. La presión atmosférica media de $1022,7 \mathrm{hPa}$, la nubosidad promedio de 2,1 octas durante las madrugadas, y la gran duración de la CAP de casi 16 horas (inicio a las 17:02 h UTC y ruptura a las 8:57 h UTC), ayuda a que los procesos de inversión térmica sean intensos, a pesar de que los flujos nocturnos erosionen parcialmente la curva de descenso de la temperatura durante la madrugada. En definitiva, el patrón sinóptico más repetido viene definido con situaciones de dorsales subtropicales, y flujos húmedos-templados sobre la Península, alternado con perturbaciones atlánticas y situaciones zonales. A pesar de que la nubosidad no es manifiesta en el sureste peninsular, sí que varias de las jornadas presentan nubosidad en la Sierra de Segura (retención en niveles más elevados), y también a un marcado flujo en niveles medios $(700 \mathrm{hPa})$, lo que ayuda a explicar que la turbulencia sea manifiesta en los niveles más elevados. 


\begin{tabular}{|c|c|c|c|c|c|c|c|}
\hline & $\begin{array}{l}\text { Evolución } \\
\text { inalterada }\end{array}$ & $\begin{array}{c}\text { Evento } \\
\text { de mezcla }\end{array}$ & $\begin{array}{l}\text { Formación } \\
\text { tardía }\end{array}$ & $\begin{array}{c}\text { Perturbación } \\
\text { alta }\end{array}$ & $\begin{array}{l}\text { Erosión } \\
\text { turbulenta }\end{array}$ & $\begin{array}{l}\text { Ventana de } \\
\text { inversión }\end{array}$ & PCAPs \\
\hline $\mathrm{HR}(>1500)$ & 50,3 & 54,3 & 75,4 & 46,1 & 43,1 & 82,1 & 36,8 \\
\hline $\operatorname{HR}(850-1500)$ & 70,4 & 57,6 & 65,2 & 53,3 & 66,8 & 81,8 & 57,2 \\
\hline $\mathrm{VV}(>1500)$ & 18,1 & 28,7 & 15,7 & 21,8 & 18,8 & 29,9 & 17,1 \\
\hline VV (850-1500) & 20,6 & 27,5 & 11,9 & 16,6 & 12,4 & 23,5 & 15,6 \\
\hline DV (>1500) & 215,0 & 93,8 & 166,8 & 175,3 & 162,1 & 337,5 & 166,5 \\
\hline DV (850-1500) & 226,1 & 195,8 & 149,3 & 185,0 & 165,3 & 168,8 & 169,8 \\
\hline HR Casas del Rey & 76,6 & 68,4 & 86,2 & 62,9 & 92,8 & 89,3 & 63,7 \\
\hline HR P. don Fadrique & 93,1 & 93,1 & 86,0 & 80,4 & 64,1 & 98,5 & 86,0 \\
\hline HR Calar Alto & 52,1 & 83,3 & 74,8 & 49,4 & 54,2 & 100,0 & 46,1 \\
\hline VV Casas del Rey & 1,9 & 3,9 & 2,6 & 1,7 & 5.3 & 7,2 & 4,9 \\
\hline VV P. don Fadrique & 4,5 & 4,3 & 3,1 & 7,3 & 4,1 & 4,0 & 8,5 \\
\hline VV Calar Alto & 20,8 & 21,3 & 16,0 & 24.4 & 23,2 & 7,0 & 31,6 \\
\hline II $(>1500)$ & $-11,2$ & $-8,0$ & $-8,2$ & $-10,3$ & $-10,3$ & $-10,9$ & $-11,3$ \\
\hline II $(850-1500)$ & $-10,7$ & $-8,8$ & $-8,9$ & $-10,9$ & $-10,5$ & $-5,3$ & $-11,1$ \\
\hline Amplitud IT & 11,1 & 6,8 & 8,3 & 6,9 & 9,3 & 15,9 & 8,6 \\
\hline Ta Mín (>1500) & $-6,0$ & $-3,0$ & $-5,5$ & $-1,3$ & $-3,9$ & $-10,9$ & $-3,6$ \\
\hline $\mathrm{T}^{\mathrm{a}} \operatorname{Min}(850-1500)$ & $-2,6$ & $-0,2$ & $-2,1$ & $-0,5$ & $-2,0$ & $-1,6$ & $-2,0$ \\
\hline Nubosidad (Murcia) & 1,6 & 1,0 & 2,5 & 5,6 & 2,1 & 5,5 & 2,9 \\
\hline Nubosidad ( $S^{a}$ Segura) & 0,3 & 4,4 & 1,1 & 2,9 & 2,6 & 4,9 & 3,2 \\
\hline Presión Atmosférica & 1022,4 & 1023,0 & 1024,3 & 1024,1 & 1022,7 & 1015,5 & 1025,5 \\
\hline Inicio CAP(h.) & $17: 43$ & $20: 56$ & 03:06 & 17:01 & 17:02 & $21: 04$ & $*$ \\
\hline Destrucción CAP (h.) & 9:09 & 9:03 & $9: 05$ & $9: 12$ & $8: 57$ & $6: 34$ & $*$ \\
\hline Duración (h.) & 15,5 & 12,1 & 6,0 & 16,1 & 15,9 & 9,4 & * \\
\hline
\end{tabular}

Tabla 3.- Características de los diferentes tipos de CAPs

\section{f) Ventana de inversión}

Incluso en las noches no ideales, con fuertes vientos o nubosidad, se pueden observar episodios de inversión de escasa duración, generalmente muy cortas en el tiempo, y cuya génesis suele ser muy rápida. Una ventana de nubosidad o un período de calma corto de 30 minutos, es suficiente para iniciar la CAP en estos episodios. En las dos jornadas analizadas (21 y 23 de enero de 2017), la duración media de la piscina llega a 9 horas y 24 minutos, con un inicio medio de la CAP a las 21:04 h UTC, mientras que la rotura se produce a las 6:34 h UTC de la mañana. Constituye el tipo de CAP más diferente al resto de las analizadas, con una humedad relativa media extraordinariamente elevada (82\%), principalmente más elevadas entre 1200 y $1300 \mathrm{~m}$ de altitud. Aquí aparecen registros higrométricos por encima del $89 \%$ en las estaciones meteorológicas situadas a $1200 \mathrm{~m}$ En niveles altos $(700 \mathrm{hPa})$ también se aprecian diferencias substanciales, con valores elevados, muy por encima de los tipos anteriormente analizados (91\%), corroborado por el 100\% de HR que registra el observatorio de Calar Alto, a 2168 m

La intensidad del viento nocturno es relativamente importante, constituyendo uno de los promedios más elevados $(26,1 \mathrm{~km} / \mathrm{h})$, con valores en niveles altos, por ejemplo, a 
$1770 \mathrm{~m}$ de altitud de $34,2 \mathrm{~km} / \mathrm{h}$ En superficie, en cambio, los valores medios de viento son relativamente bajos, entre 4 y $7,2 \mathrm{~km} / \mathrm{h}$ en los observatorios utilizados. Además, se aprecia una clara cizalladura vertical en cuanto a la dirección predominante. Por un lado, en niveles altos predomina un flujo de componente nor-noroeste $\left(337,5^{\circ}\right)$, mientras que, por debajo de $1500 \mathrm{~m}$ de altitud, aparece un marcado flujo de sur-sureste. A pesar de que los procesos de inversión térmica no son demasiado intensos $(-6,7)$, a excepción de la dolina de Monterilla con $-15,7^{\circ} \mathrm{C}$, la amplitud de inversión es la más sobresaliente del análisis, con un valor medio de $15,9^{\circ} \mathrm{C}$. El pico de La Selva registra valores de temperatura mínima de $-4,3^{\circ} \mathrm{C}$, por lo que las jornadas se desarrollan bajo la influencia de masas de aire frío. En efecto, se registran las temperaturas medias del sondeo más bajas con 2,3 ${ }^{\circ} \mathrm{C}$. Es preciso señalar, que las temperaturas mínimas registran las mayores diferencias entre niveles más elevados $(-10,9)$, y los situados por debajo de $1200 \mathrm{~m}\left(-1,6^{\circ} \mathrm{C}\right)$.

La nubosidad es imperante, con una media de 5,5 octas, y un valor medio de presión atmosférica de 1015,5 hPa, sin duda el más bajo de las jornadas analizadas. La configuración sinóptica viene marcada por una BFA frente a las costas mediterráneas españolas, con el paso en niveles altos de una vaguada que genera importante nubosidad (media de 4,9 octas según el reanálisis del modelo ARPEGE). Mientras, el flujo en niveles altos (700 hPa) apenas influye, presentando valores por debajo de $30 \mathrm{~km} / \mathrm{h}$.

\section{g) Piscinas de aire frío persistentes (PCAPs)}

En los días de invierno, algunas depresiones permanecen a la sombra de las montañas y no reciben radiación solar directa, o incluso las nieblas o nubes bajas, dificultan

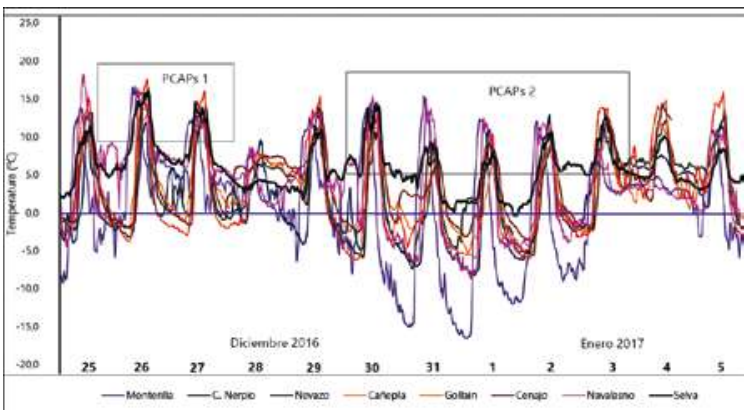

que la piscina de aire frío sea completamente destruida por el calentamiento diurno, especialmente cuando la capa de nieve está en el suelo y los árboles o cuando las nubes limitan la radiación entrante de onda corta. Por ello, son capaces de contrarrestar los efectos del

Fig. 6 Ejemplo de piscina de aire frío persistente

(PCAP) del 23 al 27 de diciembre de 2016. Referencia de La Selva (1521 m), en "atmósfera libre" representada con línea negra discontinua.

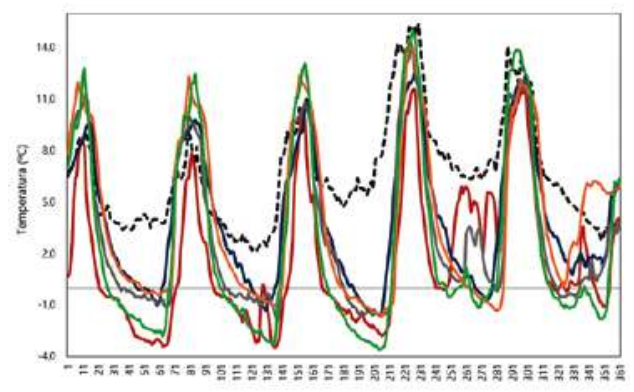


crecimiento de la capa límite convectiva, generando varios días consecutivos, donde las temperaturas máximas de valles y depresiones, no logran superar aquellas que se registran en picos de mayor altitud.

Los mecanismos que las generan son muy complejos, aunque diversos autores relacionan el parámetro del sky view factor con la propensión a padecer PCAPs más recurrentes. En el intervalo temporal del 1 de diciembre 2016 al 28 de febrero 2017 se contabilizan un total de 5 episodios generales (25-27 dic, 30 dic-3 ene, 7-8 ene, 2-4 feb y 27-28 feb), siendo el poljé colgado de Navalasno el que anota un mayor número de eventos (4), y más jornadas (10), al igual que el Nevazo. Dichos emplazamientos obtienen un valor de sky-view factor de 0,80 y 0,82, seguido de las Cañadas de Cañepla y Monterilla, con 9 y 8 jornadas respectivamente en un total de 3 episodios (sky-view factor de 0,97). A pesar, de que se concluye que los emplazamientos que registran los mayores eventos de PCAPs poseen valores de sky-view factor superiores a 0,80, la correlación existente no es demasiado elevada en el resto de observatorios $(0,44)$.

Cabe destacar que durante los eventos de PCAPs los valores higrométricos del sondeo obtienen un promedio del $49 \%$ durante las horas nocturnas, siendo especialmente bajos en niveles altos ( $28,9 \%$ a $1770 \mathrm{~m}$ de altitud). Los observatorios en niveles altos, como el de Calar Alto, arrojan valores higrométricos de 46,1\%, en clara contraposición con los fondos de valle a $1200 \mathrm{~m}$ de altitud (Puebla don Fadrique con 86,0\%). Tal y como afirma Lareau et al., (2011), los eventos de PCAPs suelen dar lugar a puntos de rocío extremadamente bajos allí donde los valores mínimos de temperatura son más bajos, tal y como ocurre en Monterilla y Navalasno. En niveles más bajos se alcanza el $65 \%$ según el sondeo ( $850 \mathrm{~m}$ de altitud). El viento, por su parte, suele ser flojo, con escasa intensidad, generalmente inferiores a $15 \mathrm{~km} / \mathrm{h}$, excepto en altitudes superiores a $1600 \mathrm{~m}$ (cercano a los $20 \mathrm{~km} / \mathrm{h}$ según el sondeo), y superando los $31,6 \mathrm{~km} / \mathrm{h}$ en Calar Alto. La dirección predominante, desde 850 a $1770 \mathrm{~m}$ es la del sur-sureste, con una presión atmosférica media de $1025,5 \mathrm{hPa}$, y cielos por lo general poco nubosos $(2,9$ octas). Durante los eventos permanentes, las intensidades de la inversión son relativamente importantes $\left(-10,6^{\circ} \mathrm{C}\right)$, la más elevada de todas las jornadas analizadas, donde casi todos los observatorios, a excepción de La Selva, superar los $-10^{\circ} \mathrm{C}$.

Las PCAPs que se generan principalmente en los sectores centrales del área de estudio, entre 850 y $1300 \mathrm{~m}$, vienen definidas por una configuración sinóptica en la que predomina una burbuja cálida en niveles medios y altos atmosféricos sobre la península (dorsal subtropical marítima), con un anticiclón británico-centroeuropeo muy reforzado $(1035 \mathrm{hPa})$. Ello da lugar a una gran estabilidad en el sureste peninsular, con un flujo a $700 \mathrm{hPa}$ muy débil y escasa nubosidad sobre el área de estudio. Esto viene a corroborar la investigación de Daly et al., (2009), quienes determinan que las PCAP son comunes durante los períodos de vientos ligeros, alta presión atmosférica y baja insolación. Además, se establecen valores térmicos más templados en niveles altos, con una temperatura media de las máximas durante los episodios de PCAPs en la Selva $(1521 \mathrm{~m})$ de $11,0^{\circ} \mathrm{C}$, mientras que en Cañada de Cañepla (1057 m) es de $10,4^{\circ} \mathrm{C}$. 


\begin{tabular}{|c|c|c|c|c|c|c|c|c|}
\hline & \multicolumn{4}{|c|}{$N^{\circ}$ Eventos } & \multicolumn{4}{|c|}{$\mathrm{N}^{0}$ Jornadas } \\
\hline & Diciembre & Enero & Febrero & Total & Diciembre & Enero & Febrero & Total \\
\hline Navalasno & 1 & 1 & 2 & 4 & 2 & 3 & 5 & 10 \\
\hline Monterilla & 0 & 1 & 2 & 3 & 0 & 3 & 5 & 8 \\
\hline Cañadas Nerpio & 1 & 1 & 0 & 1 & 6 & 2 & 0 & 8 \\
\hline Nevazo & 1 & 1 & 0 & 2 & 0 & 7 & 0 & 7 \\
\hline La Rogativa & 2 & 0 & 0 & 2 & 3 & 3 & 0 & 6 \\
\hline Cañada Cañepla & 1 & 2 & 0 & 3 & 2 & 7 & 0 & 11 \\
\hline La Risca & 2 & 1 & 0 & 3 & 3 & 5 & 0 & 8 \\
\hline Gollaín & 2 & 0 & 0 & 2 & 2 & 0 & 0 & 7 \\
\hline Barranco Cenajo & 2 & 0 & 0 & 2 & 5 & 2 & 0 & 7 \\
\hline
\end{tabular}

Tabla 4.- $\mathrm{N}^{\circ}$ de eventos y jornadas de piscinas de aire frío persistentes (PCAPs)

\section{Discusión y conclusiones}

Un aspecto tremendamente interesante es la influencia de la cobertura de nieve en las características de las CAPs y de las temperaturas mínimas registradas. En efecto, si se consideran aquellas madrugadas de piscinas frías sin cobertura nivosa en el suelo, la intensidad de inversión en Monterilla y La Risca es similar $\left(-11,7^{\circ} \mathrm{C}\right)$. Sin embargo, al analizar las madrugadas con nieve en el suelo en Monterilla (en total 7 jornadas), la diferencia de intensidad de inversión con la Risca aumenta considerablemente. Con nieve, Monterilla obtiene una intensidad de $-14,5^{\circ} \mathrm{C}$, mientras que La Risca, sin nieve, es de $-10,1^{\circ} \mathrm{C}$, incluso con jornadas como la del 21 de enero, donde la diferencia llega a $15,7^{\circ} \mathrm{C}$. De todo ello se deduce que, durante las piscinas frías con nieve en el suelo, en el caso concreto de Monterilla, se produce un descenso adicional de $4,4{ }^{\circ} \mathrm{C}$, algo menos que el observado por Dorninger et al, (2011) con descensos complementarios de 5 a $10^{\circ} \mathrm{C}$. Se determina una alta correlación entre mayor espesor de nieve e intensidades de inversión más importantes $(0,90)$. Aunque la correlación no es realmente elevada, si se aprecia una cierta relación entre la cobertura nivosa y temperaturas mínimas más bajas en Navalasno y Monterilla $(0,51)$. Además, la amplitud de inversión también es elevada, con una media de $12,3^{\circ} \mathrm{C}$.

La duración media de las CAP supera las 15 horas en los eventos de perturbación alta, erosión turbulenta y evolución inalterada, mientras que las CAP de formación tardía y ventana de inversión se sitúan claramente por debajo de 10 horas. Todas ellas, a excepción del tipo ventana de inversión, coinciden en la rotura de la piscina fría (alrededor de las 9:00 h UTC), mientras que el inicio de la misma varía entre las 17:01 (perturbación alta) y las 03:06 de la mañana (formación tardía). Además, el viento en niveles más bajos $(<1200 \mathrm{~m})$ suele mostrarse débil (entre 1,7 y $8,5 \mathrm{~km} / \mathrm{h}$ ), con valores que difícilmente pueden romper una piscina fría, mientras que en niveles altos $(>2000 \mathrm{~m})$, el viento suele mostrarse más intenso durante la madrugada (por encima de $16 \mathrm{~km} / \mathrm{h}$ ), lo que suele provocar erosiones en las curvas de descenso de las temperaturas a lo largo de la madrugada. Finalmente, los valores higrométricos en depresiones y valles de niveles bajos muestran valores elevados en la gran mayoría de los casos, mientras que, en niveles 
altos, y a excepción del tipo ventana de inversión, muestra valores higrométricos muy bajos (por debajo de 50\%), corroborando los valores que obtiene el sondeo por encima de $1500 \mathrm{~m}$ y en el geopotencial de $700 \mathrm{hPa}$.

\section{Referencias}

- Barr, S., y Orgill, M. 1989. Influence of external meteorology on nocturnal valley drainage winds. Journal of Applied Meteorology, 28(6), 497-517.

- Burns, P. y Chemel, C. 2014. Evolution of cold-air-pooling processes in complex terrain. Boundary-layer meteorology, 150(3), 423-447.

- Clements, C.B., Whiteman, C.D., y Horel, J.D. 2003. Cold-air-pool structure and evolution in a mountain basin: Peter Sinks, Utah. Journal of Applied Meteorology, 42(6), 752-768.

- Daly C., Conklin D.R., Unsworth M.H. 2009. Local atmospheric decoupling in complex topography alters climate change impacts. International Journal of Climatology, doi: DOI: 10.1002/joc.2007.

- Dorninger, M., Whiteman, C.D., Bica, B., Eisenbach, S., Pospichal, B., y Steinacker, R. 2011. Meteorological events affecting cold-air pools in a small basin. Journal of Applied Meteorology and Climatology, 50(11), 2223-2234.

- Espín Sánchez, D., García Lorenzo, R., Álvarez Ruiz, V.., y Conesa García, C. 2017. Las lluvias torrenciales e inundaciones de los días 17 y 18 de diciembre de 2016 en la Región de Murcia con particular incidencia en el área vertiente del Mar Menor. Ingeniería del agua, 21(4), 213-229.

- Gudiksen, P. H., Leone Jr., J. M., King, C. W., Ruffieux, D., y Neff, W. D. 1992. Measurements and modeling of the effects of ambient meteorology on nocturnal drainage flows. Journal of Applied Meteorology, 31(9), 1023-1032.

- LeMone, M. A., Ikeda, K., Grossman, R. L., y Rotach, M. W. 2003. Horizontal variability of 2-m temperature at night during CASES-97. Journal of the Atmospheric Sciences, 60(20), 2431-2449.

- Lareau, N. P., Crosman, E., Whiteman, C. D., Horel, J. D., Hoch, S. W., Brown, W. O., y Horst, T. W. 2013. The persistent cold-air pool study. Bulletin of the American Meteorological Society, 94(1), 51-63.

- Lareau, N. P., y Horel, J. D. 2015: Dynamically induced displacements of a persistent cold-air pool. Boundary-Layer Meteorology, 154(2), 291-316.

- Martínez Villagrasa, D., Conangla Triviño, L., Simó, G., Jiménez Cortés, M. A., Tabarelli, D., Miró Cubells, J. R., y Cuxart Rodamillans, J. 2016. Cold-air pooling in the Cerdanya valley (Pyrenees). In EMS Annual Meeting Abstracts.

- Miró, J. R., Pagès, M., y Kossman, M. 2010. Cold-air pool detection tools in the Pyrenees valleys. In 14th Conference on Mountain Meteorology.

- Neff, W. D., y King, C. W. 1989. The accumulation and pooling of drainage flows in a large basin. Journal of Applied Meteorology, 28(6), 518-529.

- Petkovšek, Z. 1992. Turbulent dissipation of cold air lake in a basin. Meteorology and Atmospheric Physics, 47(2-4), 237-245.

- Pospichal, B., Eisenbach, S., Whiteman, C.D., Steinacker, R., y Dorninger, M. 2003. Observations of the cold air outflow from a basin cold pool through a low pass. En Ext. Abstr., Vol. A, Intl Conf. Alpine Meteor. and the MAP-Meeting 2003 (pp. 153-156).

- Schmidt, E., y Beckmann, W. 1930. Das Temperatur-und Geschwindigkeitsfeld vor einer Wärme abgebenden senkrechten Platte bei natürlicher Konvektion. Technische Mechanik und Thermodynamik, 1(11), 391-406. 
Scherhag, R. 1948. Neue Methoden des Wetteranalyse und Wetterprognose. Springer Verlag Berlín, 179, 227-235.

- Steinacker, R., Dorninger, M., Pospichal, B., Eisenbach, S., Holzer, A.M., Whiteman, C.D., y Baumann, K. 2007. A sinkhole field experiment in the Eastern Alps. Bulletin of the American Meteorological Society, 88(5), 701-716.

- Vrhovec, T., y Hrabar, A. 1996. Numerical simulations of dissipation of dry temperature inversions in basins. Geofizika, 13(1), 81-96.

- Whiteman, C. D., y McKee, T. B. 1982. Breakup of temperature inversions in deep mountain valleys: Part II. Thermodynamic model. Journal of Applied Meteorology, 21(3), 290-302.

- Whiteman, C. D. 1990. Observations of thermally developed wind systems in mountainous terrain. In Atmospheric processes over complex terrain (pp. 5-42). American Meteorological Society, Boston, MA.

- Whiteman, C.D., Zhong, S., Shaw, W. J., Hubbe, J.M., Bian, X., y Mittelstadt, J. 2001. Cold pools in the Columbia Basin. Weather and Forecasting, 16(4), 432-447.

- Whiteman, C.D., Pospichal, B., Eisenbach, S., Weihs, P., Clements, C.B., Steinacker, R., y Dorninger, M. 2004. Inversion breakup in small Rocky Mountain and Alpine basins. Journal of applied meteorology, 43(8), 1069-1082.

- Zängl, G. 2002. An improved method for computing horizontal diffusion in a sigma-coordinate model and its application to simulations over mountainous topography. Monthly Weather Review, 130(5), 1423-1432.

- Zängl, G. 2005. Formation of extreme cold-air pools in elevated sinkholes: An idealized numerical process study. Monthly weather review, 133(4), 925-941.

- Zhong, S., Whiteman, C. D., Bian, X., Shaw, W. J., y Hubbe, J. M. 2001. Meteorological processes affecting the evolution of a wintertime cold air pool in the Columbia basin. Monthly Weather Review, 129(10), 2600-2613.

- Zhong, S., Bian, X., y Whiteman, C. D. 2003. Time scale for cold-air pool breakup by turbulent erosion. Meteorologische Zeitschrift, 12(4), 229-233. 